\title{
Systemic factors in patients with low-tension glaucoma
}

\author{
IVAN GOLDBERG, ${ }^{1}$ FRED C. HOLLOWS, ${ }^{1}$ MICHAEL A. KASS, ${ }^{2}$ \\ AND BERNARD BECKER ${ }^{2}$ \\ From the ${ }^{1}$ Department of Ophthalmology, University of New South Wales, Sydney, Australia, \\ and the ${ }^{2}$ Department of Ophthalmology, Washington University School of Medicine, St. Louis, \\ Missouri, USA
}

SUMMARY Nineteen patients ( 38 eyes) with low-tension glaucoma were compared with 53 subjects (106 eyes) with ocular hypertension. Comparable for age and sex, the 2 groups were assessed with respect to haematological and biochemical criteria, physical activity, and medical history. Statistical analyses of the differences between the 2 groups highlighted the importance of diastolic ophthalmodynamometry levels, prediagnosis exercise habits, cardiovascular disease status, and possibly systolic blood pressure. Patients with low-tension glaucoma suffered a higher prevalence of multiple abnormalities of these systemic factors than did their ocular hypertensive counterparts. There were no significant differences between the 2 groups with respect to the many other factors examined.

The syndrome of low-tension glaucoma consists of progressive optic nerve excavation and atrophy, with visual field loss resembling that of open-angle glaucoma, in the absence of elevated intraocular pressure. Patients with this condition can be contrasted with individuals who have ocular hypertension-raised intraocular pressure without optic nerve head damage or visual field loss. These 2 groups represent opposite extremes of the optic nerve head damage-intraocular pressure elevation spectrum. They indicate that there are factors other than the level of intraocular pressure that contribute to so-called glaucomatous visual field loss. To understand glaucomatous visual field loss better, and to improve our current therapy of patients with low-tension glaucoma, a clear delineation of these factors and their relative importance is needed. In an attempt to identify these factors we studied haematological, biochemical, physical activity, and medical historical profiles in individuals with ocular hypertension and low-tension glaucoma.

\section{Materials and methods}

The collection of data took place at the University of New South Wales, Sydney. The analysis was

Correspondence to Dr I. Goldberg, Department of Ophthalmology, Prince of Wales Hospital, High Street, Randwick 2031, New South Wales, Australia. performed in St Louis, Missouri. Patients studied met the following criteria:

Low-tension glaucoma. Intraocular pressure on no therapy of $22 \mathrm{mmHg}$ or less at all times (including following water provocation) and glaucomatous optic disc damage with or without characteristic reproducible visual field loss (defined as any one or combination of the following: nasal step of 10 degrees or greater extent, paracentral scotoma, Seidel-type scotoma, arcuate scotoma, splitting of fixation along the horizontal meridian). Patients who did not have visual field loss had had progression of optic cupping documented either by photography or by careful drawings of their discs.

Ocular hypertension. Intraocular pressure on no therapy of $24 \mathrm{mmHg}$ or more at all times without evidence of past or progressive optic nerve head damage, or visual field loss as determined by kinetic perimetry on a Goldmann-designed Haag-Streit perimeter using a modified Armaly-Rock technique. ${ }^{12}$

Excluded from the study were patients with asymmetrical ocular status, with a raised intraocular pressure secondary to a known cause (including an angle-closure mechanism), with unrelated conditions possibly responsible for the scotoma (including tumours, disc malformations, optic nerve head drusen, giant cell arteritis, retinal pathology, and neurological disease), and with a history of ocular surgery. None of the patients had received topical or systemic corticosteroids. 
Patients studied either had been attending the Eye Clinic, Prince of Wales Hospital for regular ophthalmological care, or were referred to the clinic by local ophthalmologists because they fitted the criteria defined above.

\section{CLINICAL METHODS}

At the initial visit a detailed medical history was recorded by one observer using a prearranged questionnaire. A complete ocular examination was performed by the same observer in an unmasked fashion.

As seen stereoscopically through the slit-lamp and Goldmann one-mirror contact lens, the vertical contour cup was the basis for all cup-to-disc ratio values. ${ }^{3}$ A Goldmann applanation tonometer was used for intraocular pressure measurements, and a Sisler-designed American Optical ophthalmodynamometer $^{45}$ to obtain diastolic ophthalmodynamometry levels. After the patients had been seated quietly for at least 4 minutes blood pressure readings were taken: first with the patient's arm by his/her side, and then with the arm raised so that the cuff was level with the patient's head. A specimen was obtained from each patient for urine analysis, and height and weight measurements were performed. Subsequently, in a fasting state, patients had blood drawn for a variety of haematological and biochemical investigations (see below).

Each patient returned for a fasting water provocative test: after applanation tonometry the patient drank $12-15 \mathrm{ml} / \mathrm{kg}$ body weight of cool, unsweetened tap water over 5 minutes. Thereafter intraocular pressure was measured every 10 minutes until 3 consecutive values showed that the peak intraocular pressure had been passed.

\section{STATISTICAL METHODS}

(1) The 2 groups of patients were compared with one another with respect to each factor tested by a Student's 2-sample $t$ test for continuous variables and $\chi^{2}$ or Fisher's exact probability test for discrete variables. Computations were generated by means of the Statistical Analysis System 76. ${ }^{6}$ These tests do not examine more than 1 factor at a time; therefore they do not assess the relative importance of parameters or the significance to prediction of group membership of adding factors to one another; they cannot take into account the interactions between linked-factors. Furthermore, in a study involving multiple univariate analyses, each assuming a $\mathrm{p}$ value $<0.05$ to Be significant, type I errors may occur. To overcome these problems we analysed our data as indicated in stages 4 and 5 below.

(2) In a subsidiary analysis we categorised our data on systolic and diastolic blood pressure levels and on fibrinolysis along guidelines previously used by Drance. ${ }^{78}$ This was to assess whether or not his results could be repeated in our series. For each factor the distribution of normal versus abnormal values in the 2 groups was compared by means of Fisher's exact probability test.

(3) Each of our subjects was assessed for the the presence of abnormalities in 7 vascular parameters. By means of a Fisher's exact probability test the number of low-tension glaucoma patients with 3 or more abnormalities was compared with the number of ocular hypertension subjects similarly affected.

(4) As determined by the analyses in stage 1 (above), individual variables that were significantly different between the 2 groups $(p \leq 0.05)$, those of 'borderline significance' $(0.05<\mathrm{p} \leq 0.15)$, and those of particular clinical interest were included in a Furnival-Wilson leaps and bounds algorithm as implemented in BMDP-9R in order to exclude factors whose significance was spurious. ${ }^{9}$

(5) Subsequently a more rigorous technique of multivariate analysis was used; this involved solution of the multiple logistic function. ${ }^{10}$ The values of each of the risk factors selected by means of the leaps and bounds algorithm were used to compute estimates of the coefficients of the multiple logistic function. These coefficients represented the relative contributions of all risk factors to the probability of having low-tension glaucoma as opposed to ocular hypertension, and were then used to predict this probability for each case. ${ }^{11}$

\section{Results}

Nineteen individuals met the criteria for low-tension glaucoma and 53 for ocular hypertension (Table 1). These 2 groups are comparable with respect to age, sex, and duration of diagnosis. Family history for primary open-angle glaucoma was positive in 4 patients $(21 \%)$ with low-tension glaucoma and in $7(13 \%)$ with ocular hypertension. This difference was not significant statistically $\left(p=0.35, \chi^{2}\right.$ test). All 72 individuals were white, in keeping with the relative homogeneity of the Australian population.

Patients with low-tension glaucoma demonstrated a significantly higher prevalence of both a sedentary life style and cardiovascular disease than patients with ocular hypertension (Table 2). The mean diastolic ophthalmic artery pressure was significantly lower in the low-tension glaucoma group than in those with ocular hypertension.

No significant differences were demonstrated in past history of a systemic hypotensive episode, obesity, or systemic blood pressure levels as measured with the arm at the side or with the arm 
raised (Table 2). However, as also found by Drance 8 a low systolic blood pressure (less than or equal to $120 \mathrm{mmHg}$ ) was found to be significantly more frequent among patients with low-tension glaucoma (Table 3). Such a difference between the 2 groups could not be demonstrated with regard to a low diastolic blood pressure (less than or equal to $70 \mathrm{mmHg}$ ). 'Inflow pressure' for each patient may be defined as the difference between diastolic ophthalmodynamometry and intraocular pressure. Although the patients with low-tension glaucoma had a significantly lower diastolic ophthalmodynamometry level than those with ocular hypertension, they also had a lower intraocular pressure. Mean inflow pressure in our study was $51.0 \pm 9.7 \mathrm{mmHg}$ for the low-tension glaucoma group and $56 \cdot 6 \pm 10 \cdot 7$ $\mathrm{mmHg}$ for the ocular hypertension group. This difference was not significant $(\mathrm{p}=0.07, t$ test).

Urine analysis for protein, glucose, ketones, and urine $\mathrm{pH}$ demonstrated no significant differences between the 2 groups $\left(p=0 \cdot 78, \chi^{2}\right.$ test $)$.

The fibrinolytic data were categorised by end

Table 1 Demographic data and diagnostic criteria of patient sample

\begin{tabular}{|c|c|c|c|c|c|c|}
\hline & & & Low-tension glaucoma & Ocular hypertension & $p(\mathrm{t}$ test $)$ & $p\left(\chi^{2}\right.$ test $)$ \\
\hline \multicolumn{3}{|l|}{ Number } & 19 & 53 & & \\
\hline \multicolumn{3}{|l|}{$\begin{array}{l}\text { Age in years, mean } \pm S D \\
\text { (range) }\end{array}$} & $\begin{array}{l}65 \pm 12 \\
(32-89)\end{array}$ & $\begin{array}{l}63 \pm 13 \\
(26-86)\end{array}$ & 0.56 & - \\
\hline \multicolumn{3}{|l|}{$\begin{array}{l}\text { Sex, number of females } \\
\text { ( } \% \text { females })\end{array}$} & $\begin{array}{l}12 \\
(63 \%)\end{array}$ & $\begin{array}{l}32 \\
(60 \%)\end{array}$ & - & $0 \cdot 83$ \\
\hline \multicolumn{3}{|c|}{ Mean duration of disease in months (range) } & $22(0-121)$ & $43(0-216)$ & $0 \cdot 1$ & - \\
\hline Mean IOP, $\mathrm{mmHg} \pm \mathrm{SD}$ & $\begin{array}{l}\text { OD } \\
\text { OS }\end{array}$ & & $\begin{array}{l}15 \cdot 3 \pm 2 \cdot 5 \\
14 \cdot 7 \pm 2 \cdot 6\end{array}$ & $\begin{array}{l}26 \cdot 3 \pm 8 \cdot 4 \\
25 \cdot 9 \pm 8 \cdot 7\end{array}$ & $\begin{array}{l}0.0001 \\
0.0001\end{array}$ & - \\
\hline \multicolumn{2}{|c|}{ Mean vertical contour cup/disc ratio* \pm SD } & $\begin{array}{l}\text { OD } \\
\text { OS }\end{array}$ & $\begin{array}{l}0.8 \pm 0.2 \\
0.8 \pm 0.2\end{array}$ & $\begin{array}{l}0 \cdot 4 \pm 0 \cdot 2 \\
0 \cdot 4 \pm 0 \cdot 2\end{array}$ & $\begin{array}{l}0.0001 \\
0.0001\end{array}$ & - \\
\hline
\end{tabular}

SD = standard deviation. IOP = intraocular pressure by applanation tonometry.

*Estimated stereoscopically with slit-lamp and contact lens.

Table 2 Comparison of physical activity and vascular status in patient sample

\begin{tabular}{|c|c|c|c|c|}
\hline & $\begin{array}{l}\text { Low-tension } \\
\text { glaucoma }\end{array}$ & $\begin{array}{l}\text { Ocular } \\
\text { hypertension }\end{array}$ & $p(\mathrm{t}$ test $)$ & $\begin{array}{l}p \text { (Fisher's exact } \\
\text { probability test) }\end{array}$ \\
\hline \multicolumn{5}{|l|}{ Physical activity } \\
\hline Sedentary* number (\%) & $16(84 \%)$ & $20(38 \%)$ & \multirow{2}{*}{-} & \multirow{2}{*}{0.006} \\
\hline Exercise† number $(\%)$ & $3(16 \%)$ & $32(62 \%)$ & & \\
\hline $\begin{array}{l}\text { Systemic hypotensive episode } \neq \\
\text { number }(\%)\end{array}$ & $\begin{array}{r}3(16 \%) \\
16(84 \%)\end{array}$ & $\begin{array}{r}6(11 \%) \\
47(89 \%)\end{array}$ & - & $0 \cdot 44$ \\
\hline \multicolumn{5}{|l|}{ Blood pressure } \\
\hline Systolic & $152 \pm 29$ & $164 \pm 29$ & $0 \cdot 11$ & - \\
\hline Diastolic & $92 \pm 17$ & $96 \pm 15$ & $\mathbf{0} \cdot 38$ & - \\
\hline Systolic arm elevated & $129 \pm 28$ & $136 \pm 29$ & $0 \cdot 36$ & - \\
\hline Diastolic arm elevated & $71 \pm 16$ & $73 \pm 14$ & 0.57 & - \\
\hline \multirow[t]{2}{*}{ Diastolic ODM $\S \mathrm{mmHg}$ mean $\pm \mathbf{S D} \|$} & $66 \pm 11$ & $82 \pm 12$ & 0.0001 & - \\
\hline & $66 \pm 10$ & $82 \pm 11$ & 0.0001 & - \\
\hline Cardiovascular disease present $\uparrow$ number $(\%)$ & $11(58 \%)$ & $10(19 \%)$ & - & 0.002 \\
\hline Height in centimetres, mean $\pm \mathrm{SD}$ & $164 \pm 11$ & $164 \pm 12$ & 0.91 & - \\
\hline Weight in kilograms, mean $\pm S D$ & $64 \cdot 6 \pm 13$ & $67 \cdot 7 \pm 15$ & 0.44 & - \\
\hline
\end{tabular}

*No sporting activity (activity requiring expenditure of physical energy) and walking or jogging less than 1 mile (1.6 km) per week.

†Sporting activity at least once weekly and/or walking or jogging over 1 mile $(1.6 \mathrm{~km})$ per week.

$\ddagger$ Any illnesses, injuries, or procedures resulting in profound hypotension, particularly those requiring intravenous fluids and/or blood transfusions.

§ODM = ophthalmodynamometry.

$\| \mathrm{SD}=$ standard deviation.

TCardiovascular disease of clinical importance including angina pectoris, congestive heart íailure, cardiac arrhythmias, history of myocardial nfarction. 
Table 3 Discrete analysis of patient groups

\begin{tabular}{|c|c|c|c|c|}
\hline & \multicolumn{2}{|l|}{ Blood pressure } & \multicolumn{2}{|c|}{ Euglobulin clot lysis time } \\
\hline & $\begin{array}{l}\text { Systolic } \\
\leqslant 120 \mathrm{mmHg}\end{array}$ & $\begin{array}{l}\text { Diastolic } \\
\leqslant 70 \mathrm{mmHg}\end{array}$ & $\begin{array}{l}\text { Prevein occlusion } \\
\geqslant 6 \text { hours }\end{array}$ & $\begin{array}{l}\text { Postvein occlusion } \\
\geqslant 60 \text { minutes }\end{array}$ \\
\hline $\begin{array}{l}\text { Low-tension glaucoma } \\
19 \text { patients }\end{array}$ & $4(21 \%)$ & $3(16 \%)$ & $\begin{array}{l}1(7 \%) \\
15 \text { tested }\end{array}$ & $\begin{array}{l}4(27 \%) \\
15 \text { tested }\end{array}$ \\
\hline $\begin{array}{l}\text { Ocular hypertension } \\
53 \text { subjects }\end{array}$ & $2(4 \%)$ & $6(11 \%)$ & $\begin{array}{l}0(0 \%) \\
44 \text { tested }\end{array}$ & $\begin{array}{l}7(15 \%) \\
46 \text { tested }\end{array}$ \\
\hline p (Fisher's exact probability test) & 0.04 & 0.44 & 0.25 & $0 \cdot 26$ \\
\hline
\end{tabular}

points of 6 or more hours for prevein occlusion euglobulin clot lysis time (as Drance had done ${ }^{8}$ ) and of 60 or more minutes for postvein occlusion euglobulin clot lysis time. There was no significant difference between our 2 groups for either of these 2 fibrinolytic parameters (Table 3 ).

A comparison of haematological and biochemical parameters between the 2 groups revealed a significant difference only in the case of complement factor 4 (Table 4).

Each of the 19 low-tension glaucoma patients was assessed for the presence of abnormalities in 7 vascular parameters (Table 5). Cardiovascular disease status and a history of haemodynamic crisis were based on our earlier definitions. Definitions for the other parameters are given in Table 5 . Eight of the 19 low-tension glaucoma patients had 3 or more 'abnormalities' with respect to these 7 parameters. When the patients with ocular hypertension were similarly assessed, 5 of the 53 had 3 or more 'abnormal' parameters. These distributions in the 2 groups were significantly different $(p=0.003$, Fisher's exact probability test), indicating that the prevalence of abnormal vascular parameters was higher in the low-tension glaucoma group than in the ocular hypertension group.

On the basis of the initial results the following factors were included in a leaps and bounds algorithm: (1) factors of significance $(p \leq 0.05)$ : exercise habits, cardiovascular disease status, diastolic ophthalmodynamometry, and complement factor 4 levels; (2) factors of 'borderline significance' $(0.05<\mathrm{p} \leq 0.15)$ : systolic blood pressure, fasting blood sugar levels, serum albumin, alpha 2 macroglobulin, and complement factor 3 ; (3) factors of clinical interest: haematocrit and erythrocyte sedimentation rate (considered because of their relationship with blood viscosity) and tobacco consumption, white blood cell count, total protein, euglobulin clot lysis time before vein occlusion, and a history of haemodynamic crisis.

By means of the leaps and bounds algorithm we selected the following factors for more rigorous multivariate analysis: exercise patterns, diastolic
Table 4 Haematological and biochemical parameters in patient sample

\begin{tabular}{|c|c|c|c|}
\hline & $\begin{array}{l}\text { Low-tension } \\
\text { glaucoma, } \\
\text { mean } \pm S D\end{array}$ & $\begin{array}{l}\text { Ocular } \\
\text { hypertension, } \\
\text { mean } \pm S D\end{array}$ & $p(\mathrm{t}$ test $)$ \\
\hline Haemoglobin $(\mathrm{g} / \mathrm{dl})$ & $14 \cdot 5 \pm 1 \cdot 6$ & $14 \cdot 5 \pm 1 \cdot 3$ & 0.90 \\
\hline Haematocrit (\%) & $43 \cdot 4 \pm 4 \cdot 0$ & $44 \cdot 1 \pm 3 \cdot 9$ & 0.53 \\
\hline $\begin{array}{l}\text { White blood cell count } \\
\left(\times 10^{3} / 1\right)\end{array}$ & $6 \cdot 2 \pm 1 \cdot 6$ & $6 \cdot 8 \pm 1 \cdot 7$ & $0 \cdot 19$ \\
\hline $\begin{array}{l}\text { Erythrocyte sedimentation } \\
\text { rate }(\mathrm{mm} / \mathrm{h})\end{array}$ & $9 \cdot 7 \pm 8 \cdot 4$ & $10 \cdot 3 \pm 8 \cdot 5$ & $0 \cdot 81$ \\
\hline Creatinine $(\mathrm{mg} / \mathrm{dl})$ & $1 \cdot 0 \pm 0 \cdot 2$ & $1 \cdot 0 \pm 0 \cdot 3$ & 0.95 \\
\hline $\begin{array}{l}\text { Fasting blood sugar } \\
\text { level }(\mathrm{mg} / \mathrm{dl})\end{array}$ & $93 \pm 18$ & $107 \pm 60$ & $0 \cdot 15$ \\
\hline $\begin{array}{l}\text { Blood sugar level } 2 \text { hours } \\
\text { postglucose load } \\
(\mathrm{mg} / \mathrm{dl})\end{array}$ & $119 \pm 53$ & $125 \pm 95$ & $0 \cdot 74$ \\
\hline Cholesterol (mg/dl) & $239 \pm 45$ & $240 \pm 39$ & 0.90 \\
\hline Triglycerides $(\mathrm{mg} / \mathrm{dl})$ & $130 \pm 54$ & $124 \pm 43$ & 0.60 \\
\hline $\begin{array}{l}\text { Euglobulin clot lysis time } \\
\text { prevein occlusion } \\
\text { (minutes) }\end{array}$ & $151 \pm 85$ & $127 \pm 61$ & $0 \cdot 24$ \\
\hline $\begin{array}{l}\text { postvein occlusion } \\
\text { (minutes) }\end{array}$ & $55 \pm 63$ & $45 \pm 44$ & 0.48 \\
\hline $\begin{array}{l}\text { Fibrinogen degradation } \\
\text { product titre }\end{array}$ & $3 \pm 2$ & $4 \pm 4$ & $0 \cdot 54$ \\
\hline Total protein $(g / d l)$ & $7 \cdot 1 \pm 0 \cdot 5$ & $7 \cdot 2 \pm 0 \cdot 5$ & $0 \cdot 23$ \\
\hline Albumin $(g / d l)$ & $4 \cdot 2 \pm 0 \cdot 5$ & $4 \cdot 4 \pm 0 \cdot 5$ & $0 \cdot 14$ \\
\hline$\alpha_{1}$ globulin $(g / d l)$ & $0 \cdot 3 \pm 0 \cdot 1$ & $0 \cdot 3 \pm 0 \cdot 1$ & 0.97 \\
\hline$\alpha_{2}$ globulin $(\mathrm{g} / \mathrm{dl})$ & $0 \cdot 7 \pm 0 \cdot 2$ & $0 \cdot 7 \pm 0 \cdot 2$ & $0 \cdot 36$ \\
\hline$\beta$ globulin $(g / d l)$ & $1 \cdot 0 \pm 0 \cdot 3$ & $1 \cdot 0 \pm 0 \cdot 2$ & $0 \cdot 78$ \\
\hline$\gamma$ globulin $(\mathrm{g} / \mathrm{dl})$ & $1 \cdot 0 \pm 0 \cdot 3$ & $1 \cdot 1 \pm 0 \cdot 3$ & 0.59 \\
\hline$\alpha_{1}$ antitrypsin $(\mathrm{mg} / \mathrm{dl})$ & $251 \pm 56$ & $249 \pm 61$ & 0.89 \\
\hline$\alpha_{2}$ macroglobulin $(\mathrm{mg} / \mathrm{dl})$ & $186 \pm 71$ & $221 \pm 74$ & $0 \cdot 10$ \\
\hline $\begin{array}{l}\text { Complement factor } 3 \\
(\mathrm{mg} / \mathrm{dl})\end{array}$ & $135 \pm 50$ & $116 \pm 41$ & $0 \cdot 13$ \\
\hline $\begin{array}{l}\text { Complement factor } 4 \\
(\mathrm{mg} / \mathrm{dl})\end{array}$ & $39 \pm 50$ & $49 \pm 17$ & $0 \cdot 05$ \\
\hline Immunoglobulin (mg/dl) & & & \\
\hline IgG & $1059 \pm 337$ & $1095 \pm 317$ & 0.79 \\
\hline IgM & $123 \pm 66$ & $123 \pm 66$ & 0.96 \\
\hline $\operatorname{IgA}$ & $343 \pm 194$ & $287 \pm 129$ & $0 \cdot 28$ \\
\hline
\end{tabular}

SD $=$ standard deviation.

SI conversion: Creatine $\mu \mathrm{mol} / 1=\mathrm{mg} / \mathrm{dl} \times 88.4$. Blood glucose $\mathrm{mmol} / 1=\mathrm{mg} / \mathrm{dl} \times 0.0555$. Cholesterol mmol $/ 1=\mathrm{mg} / \mathrm{dl} \times 0.0259$. Triglycerides $\mathrm{mmol} / 1=\mathrm{mg} / 1 \times 0.0113$. Total protein, albumin, and globulin, $\mathrm{g} / \mathbf{l}=\mathbf{g} / \mathrm{dl} \times 10$. 
Table 5 Distribution of abnormalities in low-tension glaucoma patients

\begin{tabular}{|c|c|c|c|c|c|c|c|}
\hline $\begin{array}{l}\text { Patient } \\
\text { number }\end{array}$ & $\begin{array}{l}\text { Low blood } \\
\text { pressure* }\end{array}$ & $\begin{array}{l}\text { Cardiovascular } \\
\text { disease present } \dagger\end{array}$ & $\begin{array}{l}\text { Glucose } \\
\text { intolerance }\end{array}$ & $\begin{array}{l}\text { Abnormal } \\
\text { fibrinolysis } \S\end{array}$ & $\begin{array}{l}\text { Haemodynamic } \\
\text { crisis }\end{array}$ & $\begin{array}{l}\text { Low diastolic } \\
\text { ODM }\end{array}$ & $\begin{array}{l}\text { Hyperlipidaemia } \\
\text { present }\end{array}$ \\
\hline 1 & - & $x$ & - & - & - & - & $x$ \\
\hline 2 & - & $x$ & - & - & $x$ & $x$ & $x$ \\
\hline 3 & $x$ & $x$ & - & $\times$ & - & $x$ & $x$ \\
\hline 4 & - & $x$ & - & - & - & $x^{\prime}$ & $x$ \\
\hline 5 & $x$ & - & $x$ & - & $x$ & $x$ & - \\
\hline 6 & - & - & $x$ & - & - & $x$ & - \\
\hline 7 & - & $x$ & x & - & - & $N T_{p}$ & - \\
\hline 8 & - & - & - & - & - & NT & - \\
\hline 9 & - & $\times$ & - & $\times$ & - & $x$ & - \\
\hline 10 & - & $x$ & NT & NT & - & - & NT \\
\hline 11 & - & $x$ & - & NT & $x$ & - & - \\
\hline 12 & - & - & - & $\times$ & - & - & - \\
\hline 13 & - & $x$ & $\times$ & NT & - & $x$ & - \\
\hline 14 & - & - & - & - & - & $x$ & - \\
\hline 15 & - & -- & - & $\times$ & - & $x$ & - \\
\hline 16 & $x$ & - & - & - & - & $x$ & $x$ \\
\hline 17 & 一 & - & - & - & - & $\times$ & $\times$ \\
\hline 18 & - & $x$ & - & 一 & - & NT & - \\
\hline 19 & $x$ & - & - & NT & - & $x$ & $x$ \\
\hline
\end{tabular}

* Systolic blood pressure $\leqslant 120 \mathrm{mmHg}$, and/or diastolic blood pressure $\leqslant 70 \mathrm{mmHg}$.

+Presence of angina pectoris, cardiac arrhythmias, congestive heart failure, or history of myocardial infarction.

$\ddagger$ Fasting blood sugar $\geqslant 120 \mathrm{mg} / \mathrm{dl}$ and/or 2-hour postglucose load blood sugar $\geqslant 160 \mathrm{mg} / \mathrm{dl}$, or presence of clinical diabetes mellitus.

$\S$ Euglobulin clot lysis time: prevein occlusion $\geqslant 6$ hours and/or postvein occlusion $\geqslant 60$ minutes.

Diastolic ophthalmodynamometry levels $\leqslant 70 \mathrm{mmHg}$.

-Serum cholesterol $\geqslant 250 \mathrm{mg} / \mathrm{dl}$ and/or serum triglycerides $\geqslant 180 \mathrm{mg} / \mathrm{dl}$ and/or abnormality of lipid electrophoretogram.

pNot tested.

ophthalmodynamometry levels, cardiovascular disease status, a history of haemodynamic crisis, systolic blood pressure, euglobulin clot lysis time before vein occlusion, fasting blood sugar level, haematocrit, and erythrocyte sedimentation rate.

Examination of these parameters resulted in a stepwise elimination of factors not contributing to the separation of patients with low-tension glaucoma from those with ocular hypertension. The final analysis included exercise, diastolic ophthalmodynamometry levels, cardiovascular disease status, haematocrit, and fasting blood sugar level. Addition of cardiovascular disease status, haematocrit, or fasting blood sugar level individually, in pairs, or all together to a model consisting of exercise level and diastolic ophthalmodynamometry did not improve prediction of low-tension glaucoma diagnosis over and above that provided by exercise habits and diastolic ophthalmodynamometry alone (Table 6).
Table 6 Multivariate logistic functions for final 5 factors in analysis

\begin{tabular}{|c|c|c|c|}
\hline & $\begin{array}{l}\text { Multivariate } \\
\text { logistic } \\
\text { function* }\end{array}$ & $p(\mathrm{t}$ test $) \dagger$ & $\begin{array}{l}p \text { (Hotelling's } \\
\left.T^{2} \text { test }\right)_{\ddagger}^{\ddagger}\end{array}$ \\
\hline Exercise alone & +4.07 & 0.0006 & 0.006 \\
\hline Diastolic ODM§ alone & +0.25 & 0.001 & \\
\hline $\begin{array}{l}\text { Exercise }+ \text { diastolic } \\
\text { ODM }+ \text { cardiovascular } \\
\text { disease }\end{array}$ & -0.99 & $0 \cdot 32$ & 0.02 \\
\hline $\begin{array}{l}\text { Exercise }+ \text { diastolic } \\
\text { ODM }+ \text { haematocrit }\end{array}$ & +0.26 & $0 \cdot 80$ & 0.02 \\
\hline $\begin{array}{l}\text { Exercise - diastolic } \\
\text { ODM - fasting blood } \\
\text { sugar }\end{array}$ & $+0 \cdot 30$ & 0.77 & 0.02 \\
\hline
\end{tabular}

*Positivity of function indicates inverse relationship between increasing value of parameter and likelihood of diagnosis of low-tension glaucoma.

tp value for that coefficient.

$\ddagger p$ Value for overall model (factors of that model pooled).

$\S$ Ophthalmodynamometry. 


\section{Discussion}

By reversing the usual association of raised intraocular pressure with visual field loss, a comparison of low-tension glaucoma and ocular hypertension groups highlights the other factors involved in the pathogenesis of optic nerve head damage.

Only 3 parameters showed a statistically significant difference between the 2 patient groups by comparison of group means-diastolic ophthalmodynamometry levels, exercise habits, and cardiovascular disease status. Considered together, diastolic ophthalmodynamometry levels and exercise patterns increased the accuracy of separation by diagnosis over either of these parameters alone. This 'prediction of fit' was not improved by the addition of any other single factor or any combination of other factors tested. Failure of cardiovascular disease status to add to the 'prediction of fit' indicates the overlap between this parameter and both diastolic ophthalmodynamometry and exercise levels.

As shown by and by others, ${ }^{78-17}$ the reduced diastolic ophthalmodynamometry with or without a lower systolic blood pressure in the low-tension group suggests that diminished optic nerve head perfusion contributed to the glaucomatous damage. Treatment of systemic hypertension may accelerate visual field loss, ${ }^{18}$ and systemic hypotensive episodes may damage the optic nerve head. ${ }^{7818-20}$

However, few people with acutely lowered blood pressure develop visual field loss. ${ }^{1315} 21$ Systemic hypertension may be protective until the onset of hypertensive vascular disease becomes a risk factor in its own right. ${ }^{22}$ Small-vessel disease in the optic nerve head and peripapillary choroid may be important in low-tension glaucoma (as it is in diabetes mellitus and ischaemic heart disease), particularly with the superimposition of a low blood pressure or acute shock syndrome. Neither systemic blood pressure nor ophthalmic artery pressure would necessarily reflect the status of these small vessels, accounting for an imperfect correlation of damage with these factors or with 'inflow pressure'.

We graded our patients' physical activities as they were prior to their ocular diagnosis so as to avoid the secondary inhibition of exertion by failing sight. As our low-tension glaucoma group had a significantly higher prevalence of systemic disease (much of which preceded the ocular diagnosis), the 'activity index' may be a reflection of this. However, exercise may be important in its own right. Confirmation is required.

The mechanism(s) by which exercise may affect optic nerve head health remain(s) unclear. Exercise tends to correct any hyperlipidaemia, defective fibrinolysis, and obesity, and to improve glucose tolerance, yet none of the differences in these parameters was significantly different between our 2 groups. Possibly physical activity assists optic nerve head microcirculation in some unknown way, perhaps by means of a prostacyclin-mediated clearance of microvascular thrombi. ${ }^{23}$ Such a mechanism is speculative. Nonetheless our investigations were not designed to detect it. At least transiently, exertion lowers intraocular pressure, ${ }^{24}$ probably by means of an induced acidosis and increase in plasma osmolarity. ${ }^{2526}$ This phenomenon does not explain our findings.

The significantly increased prevalence of overt cardiovascular disease in our patients with lowtension glaucoma compared with those with ocular hypertension is supported by Miller's study in which 10 of 31 patients with low-tension glaucoma had ischaemic syndromes outside the eye..$^{27}$ Although others have reported on the incidence of cardiovascular disease in patients with low-tension glaucoma, most have not found this significantly different from their control groups. ${ }^{782028-30}$

Drance found an increased platelet adhesiveness and/or euglobulin clot lysis time in over $60 \%$ of patients with low-tension glaucoma in both an early series of $26^{7}$ and an expanded group of $40^{8}$ such patients. Four of 7 patients with 'primary ischaemic glaucoma' (almost exclusively low-tension glaucoma) had abnormal platelet function in Spaeth's series. ${ }^{20}$ Joist and coworkers ${ }^{29}$ failed to duplicate these results. Similarly our series showed no significant difference in fibrinolytic activity between our patients with low-tension glaucoma and those with ocular hypertension.

Our failure to find a significantly higher prevalence of hyperlipidaemia in our patients with low-tension glaucoma compared with those with ocular hypertension is supported by some investigations ${ }^{19} 2930$ but not by others. ${ }^{29} 3132$ The latter 3 papers, however, used the general population as their controls. The prevalence of glucose intolerance in our study is similar to that reported previously. ${ }^{783132}$

Our study emphasises the continuing frustration of the clinician in attempting to identify factors other than intraocular pressure that are causally related to glaucomatous visual field loss. Of all of the factors investigated only prediagnostic levels of exercise, diastolic ophthalmodynamometry values, cardiovascular disease status, and perhaps systolic blood pressure appeared significant.

However, our patients with low-tension glaucoma as individuals did suffer significantly more abnormal parameters than did their counterparts with ocular hypertension. Other studies have yielded similar results. ${ }^{781922}$ This supports the concept that there 
is a complex interaction of local and systemic factors that may either maintain (in patients with ocular hypertension) or fail to maintain (in patients with low-tension glaucoma and primary open-angle glaucoma) the viability of optic nerve head tissues. Although the therapeutic value of correcting systemic abnormalities in patients with low-tension glaucoma and primary open-angle glaucoma has not yet been proved, our results appear to stress the need for the ophthalmologist as part of a medical team to detect and treat where possible whatever systemic diseases our patients with low-tension glaucoma might have. It was Lagrange who commented that often the glaucomatous eye is a 'sick eye in a sick body'. ${ }^{33}$

J. Macon Paine, BA, and Mae Gordon, PhD, tirelessly provided the statistical services demanded by this paper. Ronald M. Burde, MD, and Robert A. Moses, MD, reviewed the text.

This study was supported in part by grant EY 00336 from the National Eye Institute, Bethesda, Maryland, and by a grant from Fight for Sight, Inc., New York, New York (Dr Goldberg).

\section{References}

1 Armaly MF. Selective perimetry for glaucomatous defects in ocular hypertension. Arch Ophthalmol 1972; 87: 518-24.

2 Rock WJ, Drance SM, Morgan RW. A modification of the Armaly visual field screening technique for glaucoma. Can J Ophthalmol 1971; 6: 283-92.

3 Schwartz B. Cupping and pallor of the optic disc. Arch Ophthalmol 1973; 89: 272-7.

4 Sisler HA. Optical-corneal pressure ophthalmodynamometer. Am J Ophthalmol 1972; 74: 987-8.

5 Sisler HA. Comparative ophthalmodynamometry using scleral pressure, suction and corneal pressure units. Am J Ophthalmol 1972; 74: 964-6.

6 Barr AJ, Goodnight JH, Sall JP, Helwig JT. A User's Guide to SAS 76. Raleigh: SAS Institute, 1976.

7 Drance SM. Some factors in the production of lowtension glaucoma. Br J Ophthalmol 1972; 56: 229-42.

8 Drance SM, Sweeney VP, Morgan RW, Feldman F. Studies of factors involved in the production of low tension glaucoma. Arch Ophthalmol 1973; 89: 457-65.

9 Frane J. All possible subsets regression. In: Dixon WJ, Brown MB, eds. Biomedical Computer Programs, PSeries. Berkeley: University of California Press, 1977: 418-36.

10 Truett J, Cornfield J, Kannel W. A multivariate analysis of the risks of coronary heart disease in Framingham. J Chronic Dis 1967; 20: 511-24.

11 Miller JP, McCrate MM, Province MA, Wette R. Maximum likelihood estimation of the multivariate logistic. In: Strand RH, ed. Proceedings of the Third
Annual Conference of the SAS Users' Group International. Raleigh: SAS Institute, 1978: 363-5.

12 von Graefe A. Amaurose mit sehnerven Excavation. Albrecht von Graefes Arch Klin Ophthalmol 1857: 3: 484-7.

13 Harrington DO. The pathogenesis of the glaucoma field. Am J Ophthalmol 1959; 47: 177-85.

14 Johnson DG, Drance SM. Some studies on the circulation in patients with advanced open-angle glaucoma. Can J Ophthalmol 1968; 3: 149-53.

15 Francois J, Neetens A. The determination of the visual field in glaucoma and the blood pressure. Doc Ophthalmol $1970 ; 28$ : 70-132.

16 Harrington DO. The Bjerrum scotoma. Am J Ophthalmol $1965 ; 59$ : 646-56.

17 Heilmann K. Augendruck, Blutdruck und Glaukomschaden. Buch des Augenarztes. Stuttgart: Hept 61 Enke, 1972.

18 Drance SM. Is ischaemia the villain in glaucomatous cupping and atrophy? In: Brockhurst RJ, Boruchoff SA, Hutchinson BT, Lessell S, eds. Controversy in Ophthalmology. Philadelphia: Saunders, 1977: 292-300.

19 Chumbley LC, Brubaker RF. Low-tension glaucoma. Am J Ophthalmol 1976; 81 : 761-7.

20 Spaeth GL. The Pathogenesis of Nerve Damage in Glaucoma. New York: Grune and Stratton, 1977.

21 Jampol LM, Board RJ, Maumenee AE. Systemic hypotension and glaucomatous changes. Am J Ophthalmol 1978; 85: 154-9.

22 Laatikainen L. Fluorescein angiographic studies of the peripapillary and perilimbal regions in simple, capsular, and low-tension glaucoma. Acta Ophthalmologica $(K b h)$ 1971; 111 : 1-89 (suppl).

23 Gryglewski RJ, Korbut R, Ocetkiewics A. Generation of prostacyclin by lungs in vivo and its release into the arterial circulation. Nature 1978; 273: 765-7.

24 Stewart RH, LeBlanc R, Becker B. Effects of exercise on aqueous dynamics. Am J Ophthalmol 1970; 69: 245-8.

25 Marcus DF, Krupin T, Podos SM, Becker B. The effects of exercise on IOP: I. Human beings. Invest Ophthalmol Visual Sci 1970; 9: 749-52.

26 Marcus DF, Krupin T, Podos SM, Becker B. The effect of exercise on IOP: II. Rabbits. Invest Ophthalmol Visual Sci 1970; 9: 753-7.

27 Miller SJH. The enigma of glaucoma simplex. Trans Ophthalmol Soc UK 1972; 92: 561-84.

28 Haas JS. Low-tension glaucoma. Trans Pac Coast Otoophthalmol Soc 1962; 43: 153-60.

29 Joist JH, Lichtenfeld P, Mandell A, Kolker AE. Platelet function, blood coagulability, and fibrinolysis in patients with low-tension glaucoma. Arch Ophthalmol 1976; 94: 1893-5.

30 Walker WM, Walton $\mathrm{KW}$, Magnain $\mathrm{HN}$, Masters JB, Ward EG. Glaucoma and ischaemic vascular disease risk factors. Trans Ophthalmol Soc UK 1976; 96: 237-40.

31 Winder AF, Paterson G, Miller SJH. Biochemical abnormalities associated with ocular hypertension and low-tension glaucoma. Trans Ophthalmol Soc UK 1974; 94: 518-24.

32 Winder AF. Circulating lipoprotein and blood glucose levels in association with low-tension and chronic simple glaucoma. $\mathrm{Br} J$ Ophthalmol 1977; 61: 641-5.

33 Lagrange F. Du Glaucoma et de l'Hypotonie. Paris, 1922. 